\title{
An ITIL-Based IT Service Management Model for Distance Education
}

\author{
Shuguang Liu, Bo Dong, and Yan Sun
}

School of Electronic and Information Eng. Xi'an Polytechnic University, Xi'an 710048, China

email: L_shuguang@yahoo.com

Keywords: Distance Education; ITIL; IT Service Management Model

\begin{abstract}
In order to solve the problems in information technology (IT) services of distance education, this paper makes deep research on the IT services of School of Network Education and Information Technology Infrastructure Library (ITIL) standards, and proposes an IT service management (ITSM) model for distance education. Taking School of Network Education as an example, this study focuses on the three core processes, that is, requirements analysis and requirements change management organization structure design of distance education and application and infrastructure monitoring and management. The model provides a solution to IT services of distance education.
\end{abstract}

\section{ITIL Standards}

IT service management (ITSM) is a set of enterprise IT systems planning, development, implementation and operation of effective management is a methodology. ITSM originated in ITIL, ITIL is a set of IT service management standard library developed by the UK National Computer Board (CCTA) in 1980. Summed up in the UK in IT management, a specification for corporate IT departments to provide a set of planning, research and development, the implementation of the standard method of operation and maintenance. ${ }^{[3]}$ At present, ITSM based on ITIL standards as a globally recognized IT management best practices, and has been widely used in all kinds of national organizations and corporate IT management. Many of the world's leading organizations use ITIL to improve IT management efficiency and enhance communication between the IT and business, a large number of successful practice has shown that the implementation of ITIL IT departments can improve the operational efficiency of $25 \%$ to 30\%. ITIL in Europe and the United States and other countries to get a wide range of applications, including Procter \& Gamble, BT, AT \& T, IBM, HP, CA, Fujitsu, KPMG, EDS, Ericsson, Woodside Energy, Microsoft, HSBC and the Australian Department of Defense, etc., through the implementation of ITIL greatly improve the IT management efficiency.

The focus of the initial ITIL is that the system management, released in 2000 ITIL version 2 (ITIL V2) will focus on process management, IT service management provides the necessary process management instance. After more than two years of updates and revisions, ITIL V3 global release on May 31, 2007, and put into use, which is the largest revision ITIL since its inception, not only integrates the essence of ITIL V1 and ITIL V2, into the field of ITSM best practices - the introduction of this new service lifecycle concept, and based on the service life cycle theory regrouping ITSM best practices, including Service Strategy, Service Design, conversion services, service operators and then continuing the Service Improvement five core part, makes ITSM from the linear, static implementation of the development of multi-dimensional, feedback-oriented dynamic practice, thus promoting the combination of IT services and business development .

\section{ITIL-based ITSM Model of Distance Education Design}

ITIL standards contain the basic concepts of ITSM functions and processes, but can not be applied directly to an institution or enterprise the actual ITSM. This is because ITIL provides a theoretical framework based on best practices, does not require a company to develop an appropriate ITSM model, the model relates to the company's complex organizational structure, the level of information, 
IT application level and ITSM. Many companies (such as Microsoft, Hewlett-Packard) have developed their own ITIL models and methods. However, these models are mainly used for the company's products and services, rather than the University. Because the university in terms of organizational structure, culture, technology background and target companies are very different. This article will be based on the actual needs of the distance education and ITIL standards put forward a suitable university distance education the ITSM model (seeing Fig.1) ${ }^{[4]}$. The model will solve the main problems of distance education IT services, changing IT services, change from passive to active service. In active mode, the managers from the business perspective of distance education as a whole IT service strategy, service design and service transition, operation and maintenance personnel can make use of automated tools to actively monitor the state of the IT infrastructure and applications, early detection systems exist risks and hazards, reduce the occurrence of unexpected events, after the completion of the day-to-day work and Troubleshooting initiative for problem analysis, to assess the risk of system changes.

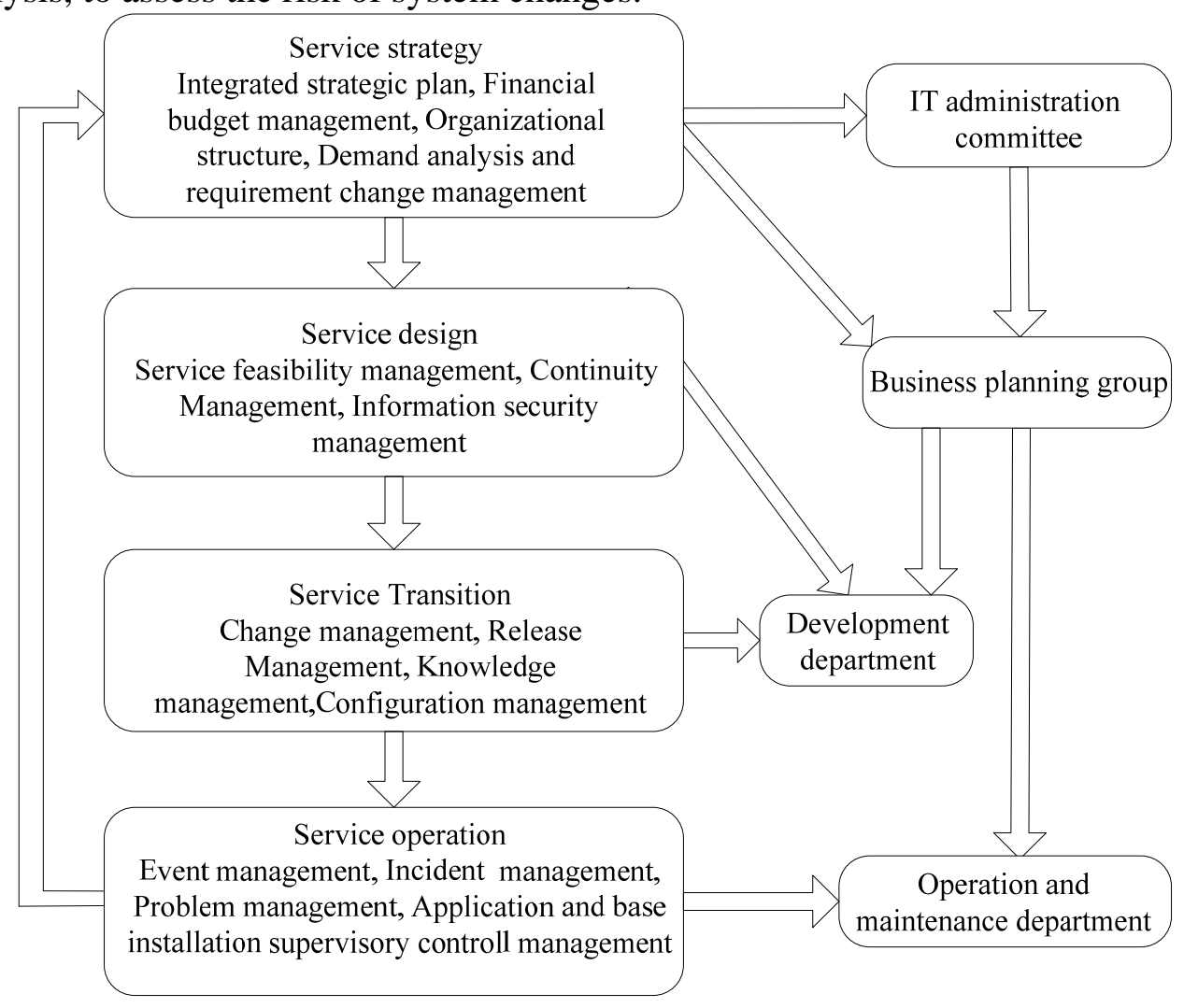

Figure 1 ITSM model of distance education

Remote education ITSM model is divided into two parts - the IT service lifecycle design and IT services organizational structure design. IT service lifecycle including service strategy, service design, service transformation and service operators the four service process to form a closed-loop system, by continuing to improve service to enhance the quality of IT services.

\section{A. Service Strategy}

Service strategy phase is to ensure the development of IT strategy and business development consistent distance education overall business objectives and management expectations. So service strategy design the overall strategic planning, budget management, organizational structure, as well as needs analysis and requirements change management four processes.

\section{B. Service Design}

The goal of the service design is the design of new or changed services into a practical application, to ensure that all aspects of the design have been taken into full consideration the design of each service should be comprehensive consideration of the relevant functional, management and operational levels. Service design stage, including service availability management, continuity management and information security management processes. Availability management is to analyze 
the user's availability requirements, to optimize the availability of IT infrastructure to ensure availability at a reasonable cost to meet the growth in demand. Continuity management goal is to ensure that the IT infrastructure and IT services after a disaster within the stipulated time to recover, to support the the school core business continuity. Information security management goal is to make IT security and business security combine to create a security risk control and response mechanism to restrict unauthorized users from using the provided IT services ${ }^{[5]}$.

\section{Service Transimission}

The Service Transition goal is to reduce known errors and changes introduced to the practical application of the risk, to provide the correct information, so that users can make decisions at the right time, in order to maintain an efficient service management processes. In distance education ITSM model conversion stage major design change management, configuration management, release management, knowledge management four processes. Change Management is responsible for the change in the service lifecycle-based configuration items, reduce risk, ensure that the change control tracking. Configuration management to manage all IT infrastructure to provide the correct configuration item information for other processes. Release management to build, test configuration items posted to the operating environment, to ensure the effective use of the service. Knowledge management to ensure that the knowledge generated in the service life cycle accumulation and sharing.

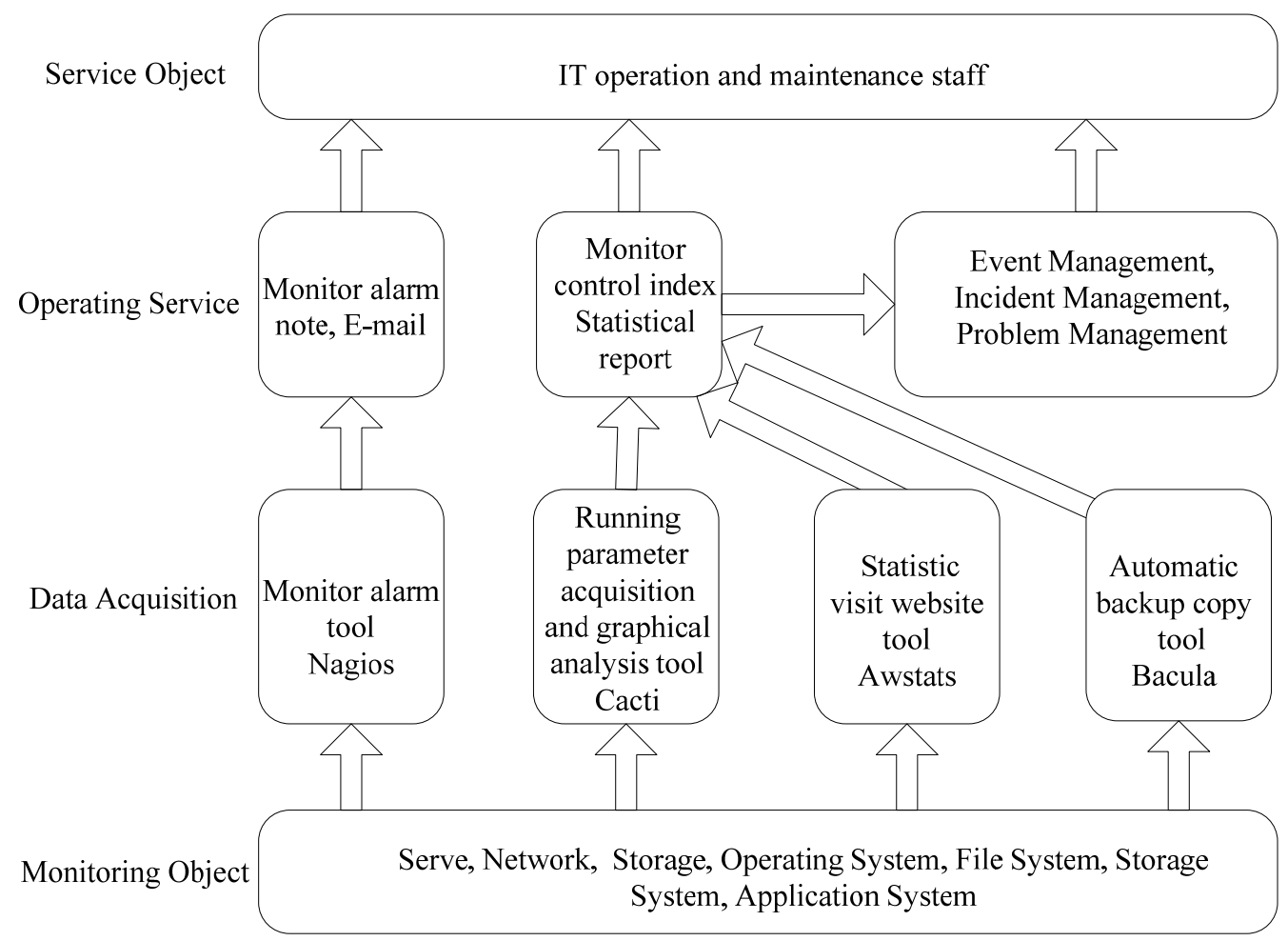

Figure 2 Model of application and infrastructure monitoring and management process

\section{Service operation}

The main purpose of the service operations through a series of day-to-day activities and coordination of the implementation of the process, to provide users with management, to achieve the established service level agreements service. The service operational phase include event management, incident management, problem management, application and infrastructure monitoring and management of four processes. Event Management is responsible for handling all failures that occurred in the day-to-day IT operations or service requests, to minimize the impact of the failure. The problem management responsible for identification of the root causes of problems and develop appropriate solutions or preventive measures to prevent their recurrence ${ }^{[5]}$. Incident Management is responsible to restore normal service operation as quickly as possible and to minimize the impact on the business, which, as far as possible, ensure quality of service and availability requirements. 
Application and infrastructure monitoring and management is responsible for weather monitoring network, hardware, applications run data and status, identify problems in advance and fast and accurate positioning.

At present, the field of open source monitoring software, using open source software to build application and infrastructure monitoring and management cost savings both for the school and the development of some of the features can be customized according to their actual needs. Application and infrastructure monitoring and management process model (seeing Fig. 2) is designed according to the needs of distance education and monitoring index system model in the model selected four open source monitoring system, Cacti, Nagios, Awstats and Bacula, these four The software monitor have different emphases.

Cacti is set based on hypertext preprocessing language (PHP), MySQL database, Simple Network Management Protocol (SNMP) and cyclic database tools (RRD Tool) development of the network traffic monitoring graphical analysis tool. Nagios is a monitoring system status monitoring and network information system, and provides alarm function via SMS and E-mail. Awstats is a simple and powerful website statistics tool. Bacula is a backup management system that allows the system administrator to manage backup, recovery and verification of computer data on the network.

\section{Conclusions}

Distance education learning, the learner can not have a time, place and space constraints, self-learning and distance education learners for serving students, most of them work during the day and study by night, the system utilization at night than during the day. The school's limited human resources can not be all-weather monitoring network, hardware, data and status of the application running, if there are problems, running around in circles, can not quickly and accurately locate the accident. Based on the needs of more than distance education, distance education ITSM model joined the monitoring and management of applications and infrastructure.

IT services according to the university network education practice in IT service issues in the current distance education, and then designed based on ITIL standards the ITSM model for distance education, and a Typical Network Education College, for example, focus on service strategy model in the process of needs analysis and requirements change management and remote education system maintenance management organization structure design, service operators in the application and infrastructure monitoring and management.

\section{References}

[1] Jiang Jingling. Innovation capability elements and Graduate Education. Chinese graduate student, 2006, (2) :44-45.

[2] Shiyong Fang, Wang Haiyong. Capacity and quality of the innovative talents training mode and practice. Scientific and technological innovation Herald. 2008, (8): 217

[3] Providing Service for Agriculture. On the basic characteristics of the innovation and quality of talent. Jiangsu Institute of Education Technology (Social Science Edition), 2000,16 (2) :1-7.

[4] Fang Guozhong, Wang Xiaojun. Innovative talent quality model based on personality traits. Northeast Normal University (Social Science Edition), 2007, 227 (3) : 106-109.

[5] Duan Xiaohong, Zhang Guomin. A Brief Discussion on the creative talents and quality characteristics. Shanxi Agricultural University (Social Science Edition), 2004, 3 (3) :223-225.

[6] Li Jiang. General education: to cultivate a sense of social responsibility and innovative people. http://www.ynufe.edu.cn/news2/NewsShowArticl.asp? ArticleID=8191. 